Historic, Archive Document

Do not assume content reflects current scientific knowledge, policies, or practices. 

$M-11-1-1$

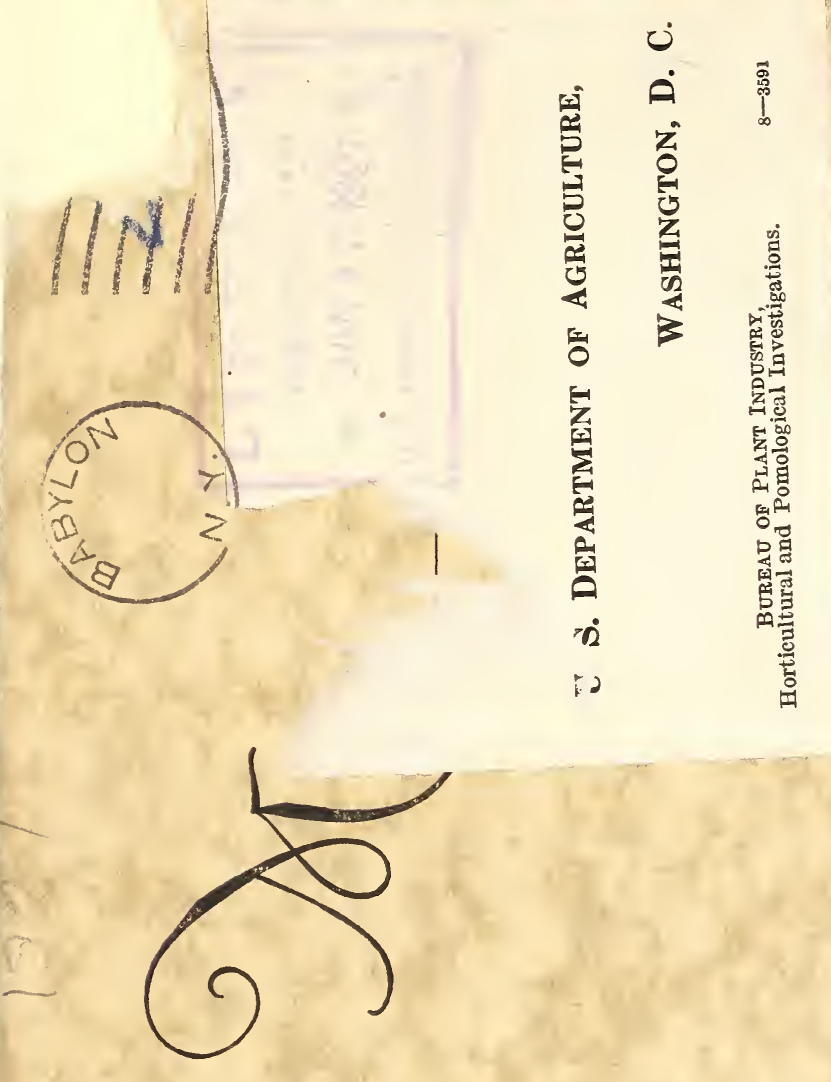

(n)

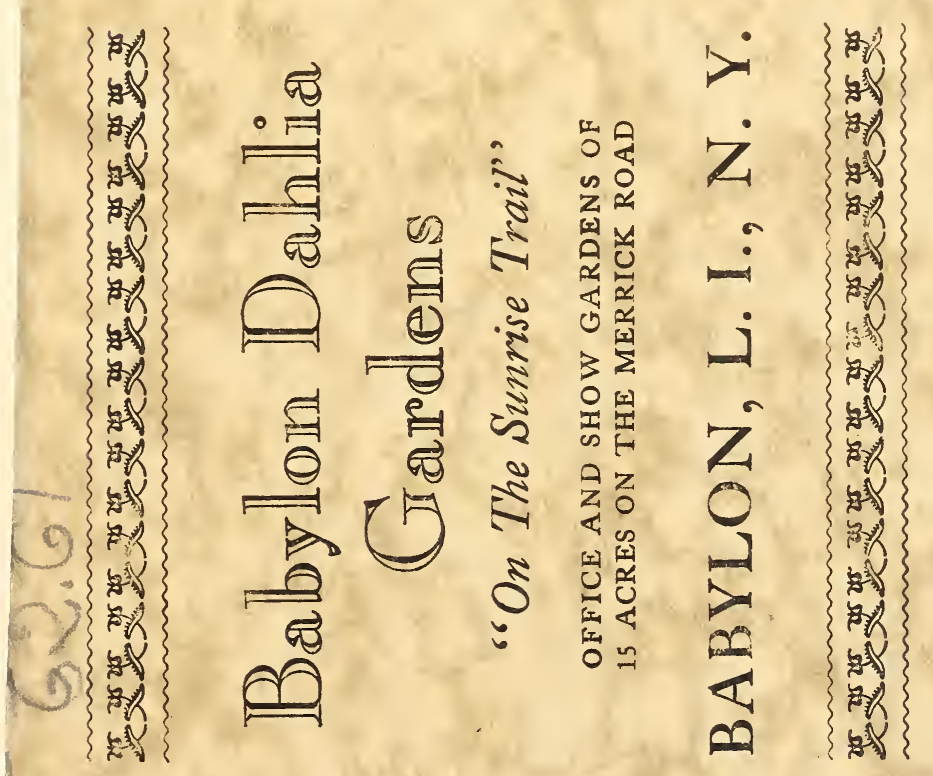


- n-

$+8$

$$
\text { 10 }
$$

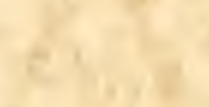

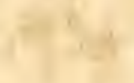

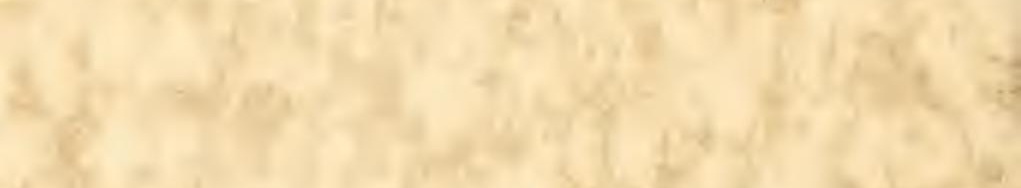

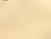

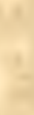

.

rach 


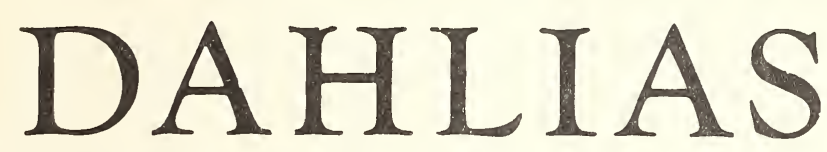

1927 Wholesale Price List

\section{To the Trade Only}

TERMS: F. O. B. Babylon, L. I., N. Y. $25 \%$ Cash with order, balance before shipping, except

to Regular Customers, or those having established credit.

\section{PROMPT SHIPMENTS-GOOD CLEAN STOCK}

Quotations are per 100 divisions of Field Grown Stock except where noted.

Ten at 100 rate.

* SUITABLE FOR CUT FLOWERS

Our Stock Guaranteed true to name is Dependable and Well Known

Prices are Within Reason and Good Business Policy

FOR THE FLORIST

DAHLIAS FOR CUT FLOWERS

The Dahlia as a cut flower is becoming more popu-

lar every year, being in bloom and at its best when other flowers are on the wane.

We list only the best.

\section{EXHIBITION DAHLIAS}

We advise a few of these fine Dahlias planted in a conspicuous part of your garden. They will attract a lot of attention and promote more sales of cut flowers. 

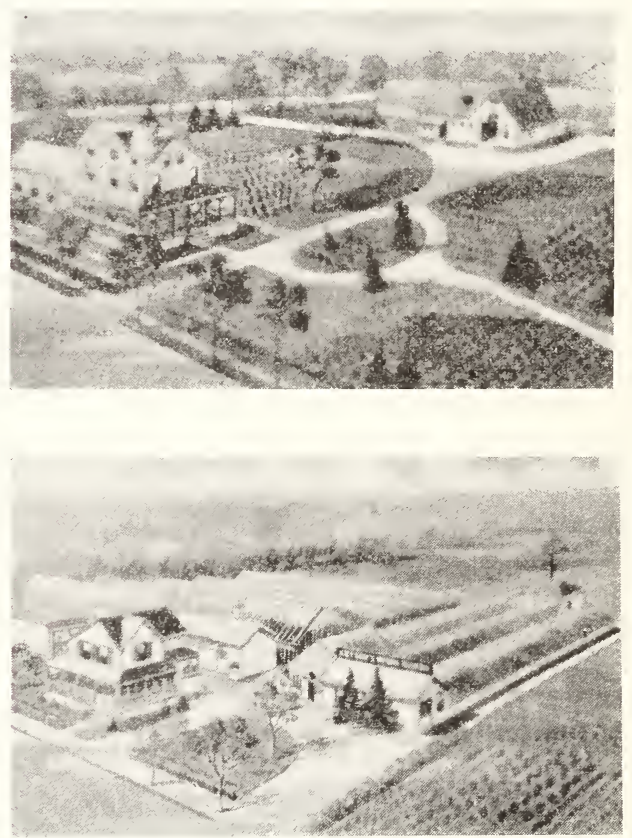

VIEWS OF OUR PLANT, SHOWING PROPAGATING AND BULB HOUSES. ALL MODERN CONSTRUCTION. 
ea. $\quad 100$

ALEX WALDIE-Creamy ground overlaid and flushed delicate pink

AMUN RA-Giant flower of coppery orange and gold tints. Distinct form. Very popular

50.00

AZALEA-Immense flower of creamy yellow, outer petals tinted pink. Very attractive. Popular

30.00

BASHFUL GIANT-Apricot and a $\mathrm{m}$ be r. Enormous flower

"BERCH VON HEEMSTEDE-Yellow, reverse rose

*BESSIE BOSTON-Fine deep scarlet clefted petals

BONNIE BRAE-Chamois suffused pink, large. Popular

* :CAMBRIA-Rich pink toning to lighter. Upright and vigorous grower, long stems. One of the very best

*CARMEN SYLVIA-Salmon, fine s'hipper...

*CATHERINE WILCOX-White tipped cerise. Very free, fine stems

$.20 \quad 15.00$

*CHALLENGE-Base yellow heavily marked garnet crimson. Early and free ................

*CHAMPAGNE - Clear champagne color. Vigorous and free bloomer on very long stems. Very large

*CHARM-Russet orange, fine stems ...........

CHIEFTAIN (Slocombe) - D e e p scarlet, fine form

CHOU (Boston)-Tyrian rose marked white. Giant flower

COPPER KING-Copper and red. Deep, full flower

*CLEMENTINA WING - Bright red, free bloomer on fine stems ................................

*CREATION-Cherry red, fine shade ......... CURFEW (Slocombe)-Fine yellow. Very large

DAKOTA-Fine shades of flame; very fine. Popular

*DOROTHY ROBBINS-Carrot buff. First class garden and cut flower

$\begin{array}{llll}\text { *DELICE_True pink; good stems } & \ldots . . . . . . . . . . . . . & .20 & \mathbf{1 5 . 0 0} \\ \text { DOAZON_Very large orange scarlet } & . . . . . . & .15 & \mathbf{1 0 . 0 0}\end{array}$

DON WILLIAMS-Soft lilac, tipped white. Very large

*DREAM-Salmon and pink, amber glow, splendid form and good keeper ................

DR. TEVIS-Very large. Salmon rose and old gold

*EARLE WILLIAMS-Very large. Red and white. Popular cut flower

EASTERN STAR—Saffron, shaded old gold. Fine type 
ELEANOR MARTIN-Mulberry. G i a $n t$ flower. Very fine

ELLINOR VANDERVEER-Lavender pink. Immense flower. Very fine

ELITE GLORY-Monster flower. Brilliant American Beauty red. Splendid $5.00 \quad 400.00$

*EL DORADO (Boston)-Pure gold, very fine $\mathbf{2 . 5 0} \mathbf{2 0 0 . 0 0}$

*ELIZABETH SLOCOMBE - Purplish garnet, vigorous grower. Very fine

*EMILIANNA (Bohrer) - Variegated red and white even marking. Flowers bring good prices on N. Y. Market. Size of lower 6 inches, fine stems and very free flowering

EMMA DeGROOT-Deep mauve, large and fine. New Holland importation

*EMPEROR-Deep rose maroon, American Beauty shade. Very large, long stems. Strong grower

E. T. BEDFORD-Enormous flower of silvery purple. Free bloomer; good stems... $\mathbf{1 . 0 0}$

85.00

*EVEQUE_Fine purple, large, on long stems

EXMOUTH GLORY-Orange yellow shading to white tips

*FLAMINGO - Delice pink, free bloomer, good stems

*FLORENCE FINGER - Deep phlox pink. Fine cutter

FRAU SCHIFF-Old favorite. Blending of apricot, orange, yellow and coral red. Very fine

*GLORIANA-Pure gold reddish glow, large

*GOODBYE DELICE-Fine true light pink. Good stem

GRACE SHERMAN-Soft pink. Fine deep built flower. Very long stems. One of the best

GRACE SPERLING-Two tone, golden buff reverse amaranth purple. Splendid cut flower, perfect stems

*GYPSY ANNA-Burnt orange, erect, long stems; very free

HERCULES - Coppery rose; fine, large flower

HIS MAJESTY_Bright scarlet, very free. Fine, large flower

*HORTULANUS FIET-Salmon pink; large

INSULINDE-Bronzy orange. Very fine....

*JACK ROSE_Crimson. Fine for cutting....

*JANE SELBY-Pinkish lavender. Fin e form, long stems

JERSEY'S BEACON-Chinese scarlet, one of the very best. Enormous flower ..........

JERSEY'S JEWELL-Mallow pink. Giant flower 
ea. $\quad 100$

*JERSEY'S BEAUTY-Most e very on e knows the sensational record made by this wonderful peer of the pinks. It stands in a class by itself. Very vigorous and clean grower on perfect stems. Cut flowers last a week. Will be universally grown. Better get started on this

*JERSEY'S KING-Carmine, good substance and stems. Coming cut flower. Better get started on this

*JERSEY'S PRIDE-Buff yellow and pink. Exhibition and cut flower

JESSIE DAY-Deep golden buff. Fine for cutting; large ..

$.40 \quad 35.00$

*JOHN MERRITT-The best creamy white we have seen. Very free bloomer on good stems

JUDGE ALTON B. PARKER-Deep buff. Large; fine for exhibition

*JUDGE MAREAN_Orange yellow red and gold. Very fine

JUNIOR (Seal) - Enormous flower, soft true lavender

*J. W. DAVIES (Boston)-Deep cerise. Very fine

*KITTY DUNLAP_Fine shade of American Beauty. Good form; long stems. Good keeper

*LADY BABBIE-Royal purple. Exceptionally free

$1.00 \quad 75.00$

LADY BETTY - Large, graceful, full flower. White suffused rose pink

LADY BLANCHE-Buff orange, with russet tints. Very fine, large flower, perfect stems. Fine for cutting or exhibition ....

LAURA WEBER (Weber) - The largest and snowiest white we ever saw. Fine stem and free flowering

LE GRAND MANITOU-W'hite and reddish purple. Speckled and blotched. Very large

15.00

"LA GROSSE BETE (Marean)-Full, perfect flower. Purple red. Fine ...................

*LE TOREADOR-Brilliant red, large flower. Fine for cutting

*LUCERO_Buff and yellow; like King of the Autumn

*MABEL THATCHER-Fine chamois; large, long stems

MAID OF THE MIST-Deep cream shading to pink. Immense deep flower. $\mathrm{Fr}$ e e bloomer. Great prize winner

MAMMOTH-Deep red. Very large; perfect form

*MARCELLA-Delice pink. Fine for florists

*MARGARET_Salmon pink, fine bouquet type, medium size. Very free 
ea. $\quad 100$

MARGARET MASSON-Silvery rosy pink. Massive, deep flower

*MERRICK (score 92 A.D.S. trial grounds)Mahogany edged lighter. Large and very free bloomer. Splendid seller. Go od stems. Very much admired

*MEPHISTOPHELES-Deep red, sometimes tipped gold. Fine for exhibition ..............

MERMAID-Yellow, very bright. Free flowering

$.60 \quad 50.00$

60.00

MILLIONAIRE-Immense flower. Lavender pink; perfect form

*MINA BURGLE_Deep red ............................

*MINNIE McCULLOGH-Soft, yellow overlaid bronzy red

$\begin{array}{ll}.10 & 6.00\end{array}$

MONS. LE NORMAND - Bright yellow splashed red

MOTHER-Mauve pink, fine form ...............

MR. DRESSELHIUS - $\mathrm{R}$ e a 1 pink, fine medium size cut flower

$.30 \quad 25.00$

$.40 \quad 35.00$

* MYRA VALENTINE (Mills) - Immense flowers; 4-ft. stems. Orange buff ......... 2.00

150.00

MRS. C. FLOOD (Murphy)_Giant flower. Pink shaded to white

*MRS. CARL SALBACH - Lavender pink. Very large, extra long stems. Flowers bring top price

MRS. DINGEE-Enormous flower. Coppery old rose, base yellow

MRS. GEO. W. ELKINS-Buff; very large, free bloomer

50.00

*MRS. I. DE VER WARNER-Orchid lavender, deep shade. Very vigorous grower on splendid stems. Free. Superior cut flower

MRS. JAY CARLISLE - DuBarry pink, shading apricot. Immense flower held upright and facing. Very fine

*MRS. JOHN T. SHEEPERS-Chamois edged pink. Very large and fine. Very popular

*MRS. J. G. CASSATT-Old rose pink, fine bloomer on good stems

*MRS. T. B. ACKERSON - White suffused and tipped carmine

*MRS. WM. WINCOTT - Best yellow cut flower we know of. Perfect form and stems. Better get started on this one ....

NIAGARA-Very large. Deep glistening red

*NOBILIS (Doolittle)—Red and white. Very large, fine flower

OSAM SHUDOW-Old rose pink. Fine, large flower

*PAPILLON (Boston)-Old rose and gold, fine bloomer

*PATRICK O'MARA-Bronzy orange. Fine cut flower 
PAUL MICHAEL_Enormous flower, pure gold. One of the very best Dahlias ever originated. Perfect for the garden

"PEACH QUEEN-Peach pink. Splendid cut flower. Very free on long stems ......

*PERLE DE LYON-Pure white. Clefted petals

*PINK JACK ROSE-Pink sport of Jack Rose. Deep shade

*PINK LADY_Fine real pink cut flower ....

*PINK PERFECTION - Like Hortulanus Fiet, only more pink

POLAR SNOW-Immense deep flower. Pure white

$3.50 \quad 300.00$

*PRIDE OF CALIFORNIA-Deep rich red, full flower. Very fine and best of this shade for cutting

12.00

PRIDE OF SAN FRANCISCO - Glowing salmon pink. Very upright

PRINCESS JULIANA-White. Very free

PRINCESS PAT-Old rose. Very fine ......

*PRINCESS MARY-Live rose pink. Most satisfactory cutter in this shade. A money maker

PURPLE MANITOU-Fine, large purple. Good form

RADIO-Red and gold. Giant flower. Very good

ROBERT SCOTT-Golden buff. Fine, deep giant flower. One of the very best ....... 3.50

ROBERT TREAT-American Beauty rose color. Very large, on fine stems ............ 2.00

ROOKWOOD_Light rosy lavender. Large and very free. One of the best ................ 1.2j

ROSE FALLON-The sensational (Harding) Dahlia of deep old gold. G i a $n$ t flower on cane stem ..................................6.60

ROSA NEL_Bright cerise rose. Very large. Perfect form

* ROSE ACRE-Deep red tipped and shaded white. Brought good prices as cut flowers. Fine keeper and shipper

:SAGAMORE-Apricot buff. Fine cut flower 2.50

*SAMSON-Variegated red and yellow. One of the very best

SEQUOIX GIGANTEA-Very large yellow. Fine habits. Vigorous

SENORITA-A real giant. Deep velvety crims on

*SHANNON-Golden chamois buff. Very good

*SHELIKOF-Very dark maroon. Very fine

SHOWER OF GOLD-Brilliant yellow and apricot, very free

SNOWDRIFT-H u ge flower of perfest form. Pure white; very popular. Dwarf grower; vigorous 
ea. 100

*SUNNY JERSEY-Lemon yellow suffused gold. Very free on fine stems. Good cut flower

$2.50 \quad 200.00$

*SYLVIA DICKEY-Light phlox pink. Very free on fine stems. Splendid for cutting

TANFORAN_Deep scarlet; very large, fine stems

$.25 \quad 20.00$

$1.00 \quad 75.00$

TOMMY ATKINS (Boston)-Very brilliant scarlet. Fine, large flower; good stem ....

TRENTONIAN-B r o w n coppery bronze. Immense flower on fine stems. Very fine 3.50

$.75 \quad 60.00$

*ENUS-White suffused lavender. Coming cut flower. Very popular

$.25 \quad 20.00$

*VERDUN-Yellow and scarlet. Very bright. Fine cut flower

$.75 \quad 60.00$

*W. E. COOPER (Boston)-Real live pink. Stiff stem. Free

$.75 \quad 60.00$

*WHITE DOVE - Here we have the best snowy white florist's Dahlia. Splendid for making up

$.20 \quad 15.00$

*WINFIELD SLOCOMBE-Deep, full flower of golden buff shading darker fine autumn shades. Long stems. Good bloomer and keeper

WM. SLOCOMBE-V e ry large and free. Canary yellow

WORLD'S BEST WHITE-Creamy white. Large, deep flower of fine form and stems

WORLD'S WONDER-Deep buff. V e r y free bloomer on long stems ...

$1.25 \quad 1.00 .00$

$.35 \quad 30.00$

YELLOW CHRYSANTHEMUM - Splendid light yellow. Very large and wonderful form

ZEUS-Bright red tipped golden yellow. Stunning effect

\section{CACTUS and HYBRID TYPES}

AMBER GLOW-Very large, pure amber....

ea. 100

$.60 \quad 50.00$

BABA BOTT-Improved Helen Durnbaugh. White suffused pink. Good seller

BABYLONIAN-Wine crimson. The best in its color of this type. Very large, free, and fine form. Splendid stems...

BALLET GIRL-Buff orange tipped white, red striped, white and solid buff on same bush. Splendid type and large. Popular

*BIANCA-Rosy lavender. Very free $.60 \quad 50.00$

BILLIE BURKE-Straw yellow, edged rosy lavender. Very large

$.20 \quad 15.00$

$.40 \quad 35.00$

BOB PLEUSE-Deep crimson, tipped white. Very free 1.25100 .00

BONNIE G.-Real pink, large flowers ....... $\quad .50 \quad \mathbf{4 0 . 0 0}$

*BRIDE'S BOUQUET - P u r e glistening white, fine for cutting 
CANADIAN QUEEN - Enormous flowers, pink and white

*CINDERELLA (Boston)-Very large buff and orange pink flush. Upright

*COLOSSAL PINK (Peacock)-Clear pink and white

*COUNTESS OF LONSDALE-Salmon on rose. Fine garden and cut flower Dahlia

DADDY BUTLER - Carmine rose shaded lighter. Large and attractive ................. 1.00

*DOROTHY DURNBAUGH-Very large, upright flower. Salmon pink ..................... $\quad .40$

DUCHESS OF MARLBORO - $\mathrm{P}$ in $\mathrm{k}$ and white. Very shaggy, pointed petals .....

EDITH SLOCOMBE-Crimson purple. Fine shade. Very attractive

ELSIE DANIELLS-Bluish lavender, center white; beautiful

EL GRANADA-Vivid orange, creamy reverse. Very large

ELSIE OLIVER (Boston) - Exceptionally large salmon and pink; fine form, upright $\mathbf{1 . 0 0}$

75.00

*EMMA MARIE-Pink shading to white centre. Fine cut flower

*ESTHER HOLMES-Fine lavender; upright stems. Very free

*ETENARD DE LYON-Fine purplish lavender. Very free, large and good stems

F. W. FELLOWS-O $r$ a n g e terra cotta. Popular

FRANCIS LOBDELL (Waite)-M a $110 \mathrm{w}$ pink. Fine type ..................................... 1.00

75.00

* GAY PAREE-Golden bronze, suffused red. Very fine; upright

50.00

GEE WHIZ - Buff and salmon. Immense flower

50.00

GEORGE WALTERS-Very popular. Salmon, gold and pink; free flowering .........

GOLDEN EAGLE-Deep buff

*GOLDEN PRINCE-Perfect golden yellow of large size. Vigorous grower

(1)

GOLDEN WAVE-Yellow. Large, fine form for exhibition

GLADYS SHERWOOD-Very large white GOLDEN GATE-Golden amber

ISLAM PATROL-Very showy. Deep velvety scarlet tipped and flushed gold ........

*J. B. RIDING-Orange and apricot. Large and free bloomer. Straight stems ..........

*KALIF-Scarlet. Large. Can't be beat as yet

*LA FAVORITA-Fine deep orange; large....

LORAINE WOERNER-Creamy yellow and salmon pink. Large and full

MARJORIE CASTLETON_Pink and white. medium size; popular 
*MARY DOUCET-Pink and white. Perfect stem. Fine

MONTAUK-Salmon pink. Full flower, good stem and growth. Our own introduction

MT. SHASTA-Delicate coral pink; very large

* MRS. F. W. STREET-Crimson t i p ped white. Very showy

*MRS. EDNA SPENCER-Delicate pinkish lavender; upright

MRS. GEORGE WAGSTAFF-Spinnel red. Very free on splendid stems; large

MRS. HAPPIE BEDFORD - Exhibition flower of immense size; perfect form. The best of this type and color

*MRS. H. J. JONES-Red and white. Very popular cut flower

MRS. WARNAAR-White flushed pink; very large. Great favorite

NIBELUNGENHORT - Salmon rose; fine old favorite

NICHU_Clear light yellow; large, f ull flower

* RED CROSS—Scarlet and gold. Very large and fine

* RUTH FORBES_Mauve pink. Large, fine stem

SA YVILLE-Straw yellow, tinted red; upright

SHIRLEY BROWN-Old gold, tinted salmon. Very large and fine

*SONNENGOLD-Golden orange. Very fine, long stems

SOUTHWARD HO-Very large flower and deep. Same form as Kalif; deeper scarlet and tipped white. Attractive; will be good seller. Our own introduction .........

*SUNBRITE-Yellow and red; similar to Red Cross. Very free; good stems .........

SUNKISS-Canary yellow, flushed at edge with rosy salmon. One of the best ..........

SUNSET GLOW (Peacock)-Red and gold; very free. Medium sized flower; fine for cutting

VIOLETT A-Petunia violet. Splendid acquisition

*VIOLA PHILLIPS-One of the best ever introduced of this type and color; salmon and mauve. Fine keeper

WASHINGTON CITY_Fine white flower, of good depth. Popular

W. B. CHILDS-Deep maroon; good ..........

WIZARD OF OZ-Enormous flowers, amber pink and salmon. Splendid exhibition type

*YELLOW KING-Very large, full flower; sulphur yellow, tipped lighter 


\section{PEONY TYPE}

*AURORE-Large flower of orange buff; good stems and keeper. Very fine ..........

**BILLIONAIRE-Orange buff; very large....

*CALIFORNIA — Canary yellow; good ..........

CAMILLE FRACHON_Glistening garnet; very attractive

ea. 100

$.20 \quad 15.09$

$.50 \quad 40.00$

$.25 \quad 20.00$

CARDINAL MERCIER - Immense flower of sparkling salmon, rose and pink ........

CHATENAY - Salmon pink

$.50 \quad 40.00$

$.75 \quad 50.00$

$.15 \quad 10.00$

DREAMLIGHT-Rose pink, b a s e golden yellow. Large and free

$.75 \quad 60.00$

FANTASTIQUE-Variegated; white striped purple. Very odd

$.20 \quad 15.00$

GEORGE MASTICK-Deep crimson; very large and free

$\begin{array}{ll}.30 & 25.00\end{array}$

GLORY OF NIJKERK-Purple violet; very free

20.00

GORGEOUS-Very large; scarlet and gold

LADY ALLA-Bright scarlet; resembles a poinsettia

$.75 \quad 50.00$

$.20 \quad 15.00$

$.25 \quad 20.00$

*LO XIII-Canary yellow; very large ...... pink; very free. Fine for decoration .....

*MADONNA-White; large and very free....

MME. BIJSTEIN-C l e a r light lavender. Very graceful

MEYERBEER-Very large flowers of fine maroon purple. Popular ............................

MOONFACE-Primrose yellow. Very free

OREGON BEAUTY-Oriental red. Fine garden dahlia

PEARL RUGGLES-Carmine suffused pink, white base. Very artistic .............................

PITTI SING-Scarlet and gold; fine substitute for Geisha. Very brilliant ............

*REGINA-Salmon. Very free, long stems. Fine for cutting ...............................................

RODMAN WANAMAKER (Peacock) Enormous flower, maize yellow tinted on edge old rose

$.40 \quad 35.00$

$.20 \quad 15.00$

$.20 \quad 15.00$

$.30 \quad 25.00$

$.15 \quad 10.00$

$.15 \quad 12.00$

$.30 \quad 25.00$

$.40 \quad 35.00$

$.30 \quad 25.00$

$2.50 \quad 200.00$

SULPHEREA-Light yellow; shaped like chrysanthemums; fine

$\begin{array}{ll}.30 & 25.00\end{array}$

*SWEETHEART'S BOUQUET-Lovely, deep pink. Large, long stems ........................... $\quad .30$

UNCLE SAM-Immense golden apricot ..... 1.25

25.00

100.00

\section{BALL or SHOW TYPE}

*A. D. LIVONI-Shell pink. Old favorite and still popular

*ACQUISITION-Deep lavender; fine ........... .20

*ARABELLA-Pink and buff; an old favorite

*AUGUST MEGAR-Pansy purple; perfect form

*CUBAN GIANT-Deep crimson; large $\ldots . . . \quad .15 \quad \mathbf{1 0 . 0 0}$

DELIGHTED-Very large white .................. $.15 \quad 10.00$ 
*ELSIE BURGESS-White tipped and suffused lavender. Fine for design work. Very long, straight stems

*GOLD MEDAL-Scarlet suffused gold ........

*JOHN BENNETT-Perfect form; deep buff and red

*JOHN WALKER-White, good form ..........

*MAUD ADAMS-Mauve pink and white very free

.158 .00

*MRS. J. P. SMITH-Cherry red, perfect form; large and free

$.20 \quad 15.00$

PURPLE GEN'L MILES-True purple; very large, fine form

*RED DUKE-Deep red; fine form. Very free, on long stems

*STORM KING-White, very free ............... $\mathbf{. 1 5} \mathbf{1 0 . 0 0}$

*SURPASSE COLOSSE-Old rose; g o o d habit. Very good

VIVIAN-Dark magenta flushed cerise. Large; popular

*WHITE SWAN_Most reliable white for cutting in this type

*W. H. WILLIAMS--Maroon red; good form, fine cutter

\section{COLLARETTES and SINGLE TYPE}

MAURICE REVOIR-Crimson, with white collar

ROYALE-Deep crimson purple, white collar. Very decorative

AMI BARTLETT_Scarlet, purple foliage. Fine for border and bedding

\section{POMPOMS}

ACHILLES-Lavender pink

ALEWINE-White ground, flushed lavender

AMBER QUEEN-Amber and orange ......

ARIEL-Buff tinted orange ............................

BELLE OF SPRINGFIELD — Red; very small

BOBBY-Plum

CATHERINE-Clear yellow

DARKSOME-Deep maroon

FASCINATION_Fawn pink ...................... .15

GANYMEDE-Fawn ….................................... $\quad .20$

GLORIA-Pink and maroon ............................ .15

KLEIN DOMITEA-Orange buff ................... .15

LITTLE BEAUTY_Shrimp pink ..................

LITTLE DOROTHY-Buff and white .......... .15

LITTLE BESSIE-Creamy white ......................15 


\section{PLANTS FOR I927}

P.S.-We have completed two new propagating houses, $21 \times 100$ feet each, to take care of our increasing business in plants. We are in a better position than ever to give you service.

\section{DAHLIA GREEN PLANTS}

Not just ordinary rooted cuttings, but good strong plants, grown cool, well rooted, ready to grow right on, produce exhibition flowers and tubers free from disease. We have many repeat orders and our orders for them are increasing each year. Try them and be convinced.

Packed right to ship long distance and guaranteed to arrive in good condition.

All orders for less than $\$ 5.00$ add $5 \%$.

ea. doz.

ALICE M. LONGFELLOW-(F.\& M.) ....... 1.50

AMUN RA-(Seal) ........................................ .40 4.00

*AMBASSADOR_(Broomhall) $\quad$........................ $50 \quad 5.00$

BERTHA JOST-(Jost) ...................................50 $\quad .50 \quad 5.00$

*BABYLONIAN (Babylon)_Deep burgundy red, shaded velvety black. Splend id grower and bloomer on fine stems

BASHFUL GIANT-(Marean) .......................50 $\quad .500$

*BLACK JACK-(Dressler) ............................ $\quad .75 \quad 7.50$

BLUE LADY_(Seal) ................................... .75

BOSTON WHITE-(Murphy) ......................... $\quad .75 \quad 7.50$

*CALIFORNIA SUPERBA-(Boston)

*CAPT. JOHN (Capt. John Howell)_Clear orange buff. Large flower on perfect stems

*CARMELLO (Callahan)-L a $r$ g e flowers held upright. Amaranth pink; very fine new one

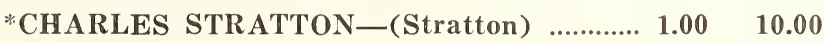

*COPPER KING-(Marean) .......................... $1.00 \quad 10.00$

*CIGARETTE-(Boston) .................................. .75 7.50

*CHAMPAGNE—(Boston) ….......................... $\quad .60 \quad 6.00$

CITY OF LAWRENCE-Reed .................... 1.50 15.00

*CLARA FINGER_Finger ............................. $1.00 \quad \mathbf{1 0 . 0 0}$

CARUSO-(Stillman) $\quad$..................................... $1.00 \quad 10.00$

DARK DAYS-(Marean) ............................. 1.00

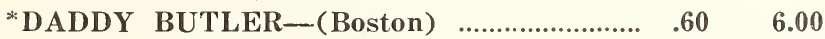

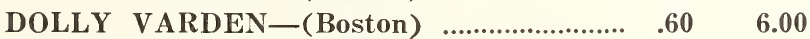

*DR. E. H. BARTLEY (Schwartz)—Gigantic flower. Orchid lavender. Can easily be grown 12 inches; long stems ....................... $3.00 \quad 30.00$

*DON WILLIAMS-(Doolittle) ..................... $1.00 \quad 10.00$

EAGLE ROCK BEAUTY (Broomhall) ........10.00 100.00

EAGLE ROCK SUNSHINE (Broomhall)... $3.00 \quad 30.00$

ESTHER HUNT-(Boston) ............................ 1.00 10.00

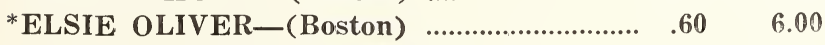

*EASTERN STAR-(Maytrott) ..................... 1.00 10.00

*ELITE GLORY - (Kennedy) ......................... $3.00 \quad 30.00$ 


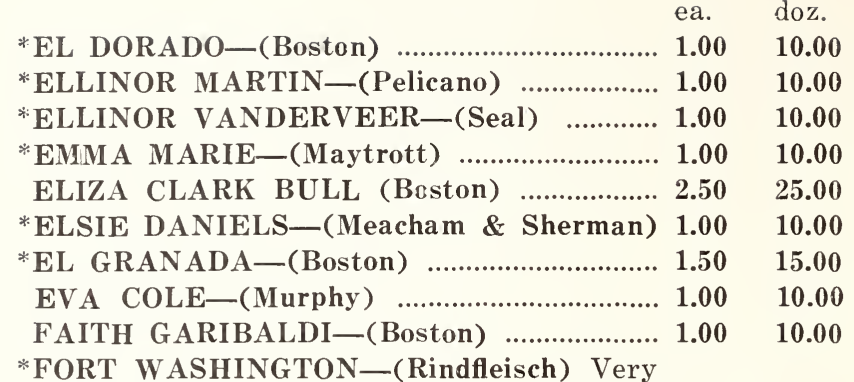

*FORT WASHINGTON-(Rindfleisch) Very large blackish maroon; perfect form, fine stems

GLORY OF SUNRISE TRAIL-(Babylon) Maise yellow edged violet; giant flower. Splendid stem. A seedling of the famous Sunkiss. Will be one of the best sellers GOLDEN FLEECE-(Seal)

*GRACE SHERMAN - (Brock) Improved Cambria, larger and blooms more freely. Very fine

GRACE SPERLING-(Babylon) Two-tone buff, reverse amaranth purple. Very free flowering; perfect form

*HIS MAJESTY-(Boston)

*HERALD TRIBUNE ELITE-(Kennedy).... $1.00 \quad 10.00$

HUGO SCHUSTER - (Obert) Decorative seedling of U. S. A. Very free flowering; fine form

*ISLAM PATROL-(Davies)

*JERSEY'S BEAUTY—(Waite) .................... .35

3.50

"JERSEY'S BEACON-(Waite) ..................... 1.00

JERSEY'S IDEAL-(Waite)

$2.00 \quad 20.00$

*JERSEY'S JEWELL-(Waite)

$1.00 \quad 10.00$

*JERSEY'S RADIANT-(Waite) ................... 1.00

KING SOLOMON-(Andrews) ...................... 1.50

*KING TUT-(Seal) ….................................... 2.00

10.00

15.00

20.00

*LADY BLANCHE-(Babylon) Straw yellow overlaid russet. Fine exhibition and cut flower

*LA GROSSE BETE-(Marean)

6.00

*MARGARET MASSON-(F. \& M.)

MARION WELLER-(Marean)

1.00

10.00

$1.50 \quad 15.00$

*MARIPOSA-(Boston)

7.50

*MERCEDES-(Boston)

7.50

*MORDELLA-(King)

*MRS. DINGEE-(Brock) Deep salmon rose underlaid yellow; very large and $\mathrm{free}$ flowering. Splendid

*MRS. HAPPIE BEDFORD - (Brock) The largest, best formed and finest yellow $\mathrm{H}$. C. we have seen. Stems slightly pendant $\mathbf{1 . 5 0}$ 
ea. doz.

*MRS. JAY CARLISLE-(Babylon) Grenadine pink. Very large, fine bloomer. Vigorous grower

*MYRA VALENTINE_(Mills) ................. $1.00 \quad 10.00$

*NICHU-(Boston) .................................. $1.00 \quad 10.00$

*OLIVE REED-(Seal) ........................... $1.00 \quad 10.00$

OYSTER BAY BEAUTY-Rich dark glowing red. Very free, fine stems. Does not burn. As large as Elite Glory ............... 2.00

*PEACOCK-(Peacock) .............................. 1.00

*POLAR SNOW-(Marean) ......................... 1.00

POP STEWART-(F. \& M.) .................... 1.50

*PAPILLION-(Boston) …........................ .75

*PENATAQUIT-(Howell) ............................60

*PRIDE OF SAN FRANCISCO-(Pelicano) $\quad .75$

QUEEN OF THE GARDEN BEAUTIFUL

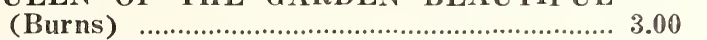

*RADIO-(Sampson) $\quad$................................. 1.00

*ROBERT SCOTT-(Brock) ...................... 2.50

*ROBERT TREAT-(Mueller) .................. 1.00

ROMAN EAGLE-(F. \& M.) ................. 1.00

*ROSE FALLON-(Harding) ...................... 2.50

*RODM AN WANAMAKER-(Peacock) .... 1.00

SANHICAN GEM-(F. \& M.) ................ 1.50

SANHICAN MAGNATE-(F. \& M.) ....... 1.50

SANHICAN MONARCH-(F.\& M.) ........ 1.50

SANHICAN ROSE-(F. \& M.) ................ 1.00

*SHIRLEY OLIVER-(Seal) ..................... 1.50

*SOLE MIO-(Lohrmann) Splendid yellow.

Very large, fine form and free flowering.

One of the best .................................... 1.50

*SUNNY JERSEY-(Maytrott) ................. 1.00

*SAGAMORE-(Badetty) …....................... 1.00

*SUSAN TEVIS-(Boston) ........................ 1.00

SISKIYOU-(Broomhall) ......................... 1.50

*SHUDOW'S LAVENDER-(Boston) ........ . .75

TALISMAN_(Boston) …........................... $\quad .75$

*TRENTONIAN-(F. \& M.) ....................... 1.00

*UNCLE SAM-(Meacham \& Sherman) ..... $\quad .75$

VINELAND-(Maytrott) …....................... 1.00

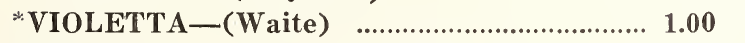

*WIZARD OF OZ-(Doolittle) ................... $1.00 \quad 10.00$

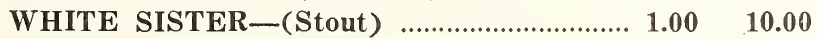

*WORLD'S BEST WHITE-(Murphy) ....... $\quad .75 \quad 7.50$

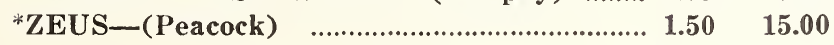

DAHLIAS FOR COUNTER TRADE

\begin{tabular}{|c|c|}
\hline 100 & 1000 \\
\hline MIXED ................. & $\$ 55.00$ \\
\hline MIXED FANCY & 70.00 \\
\hline MIXED FANCY EXTRA .................................... & 100.00 \\
\hline 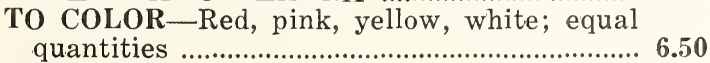 & 60.00 \\
\hline
\end{tabular}




\section{Memorandums}




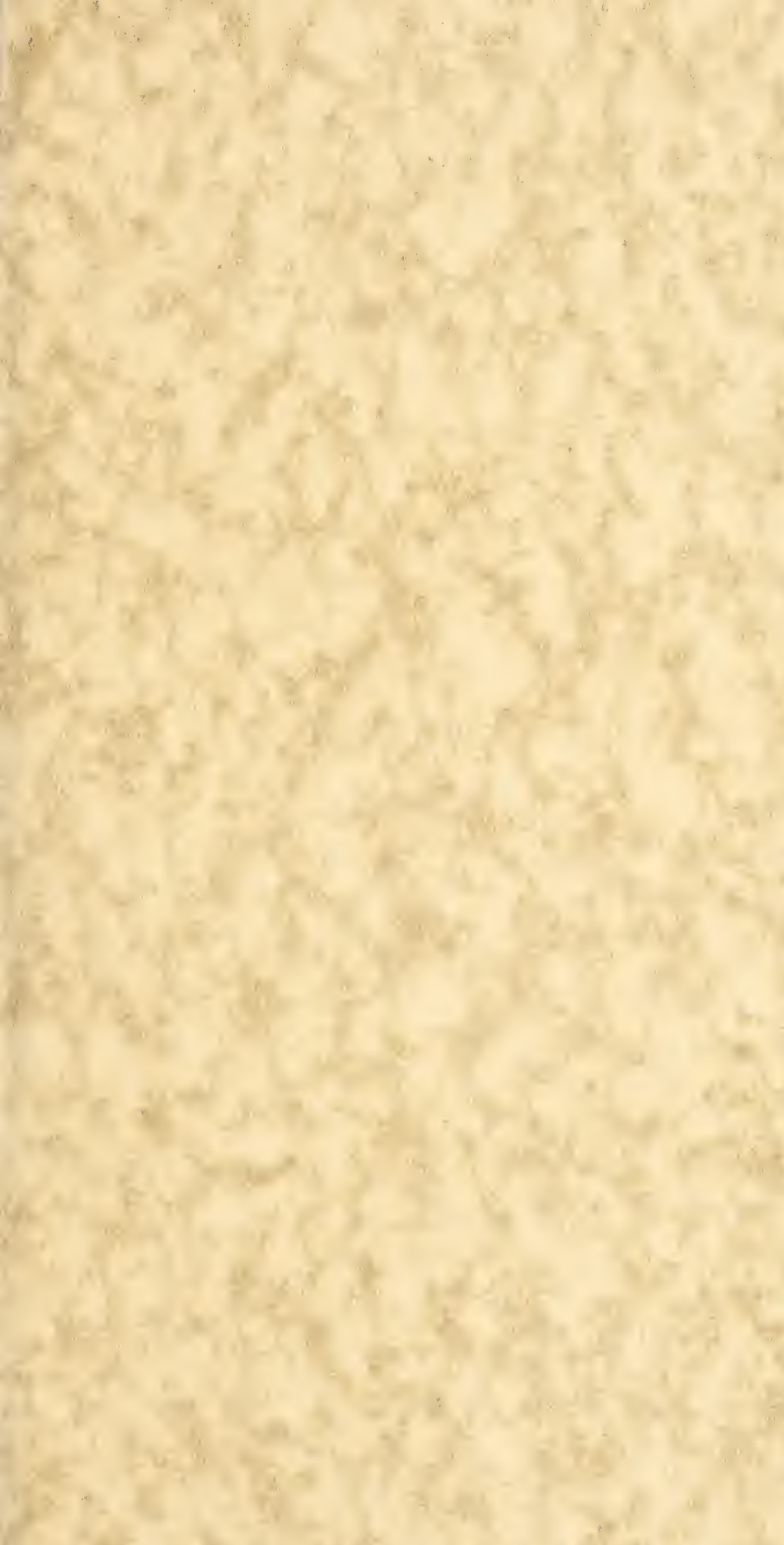




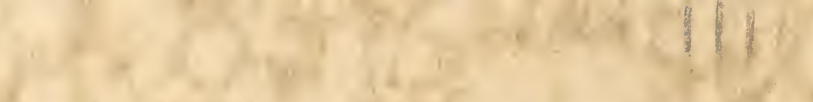

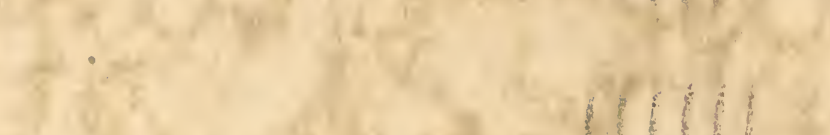

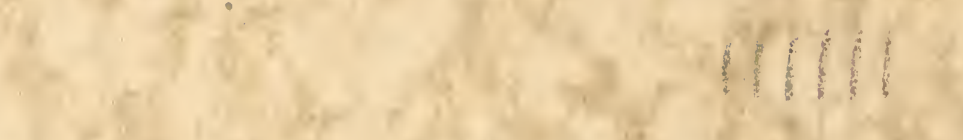

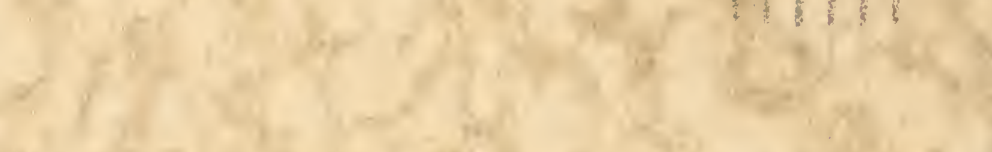

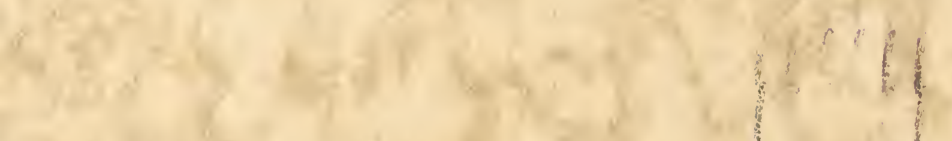

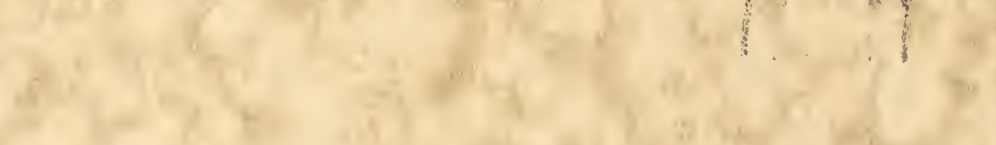

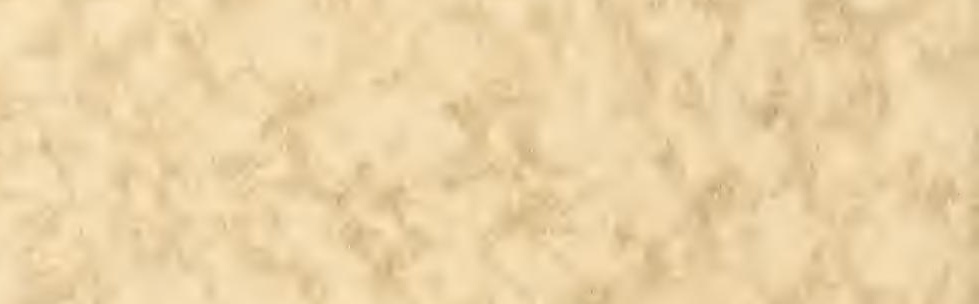

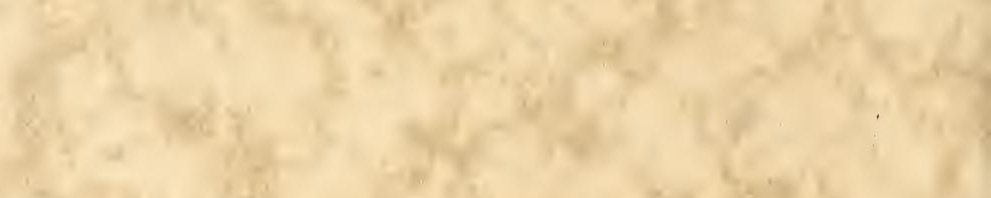

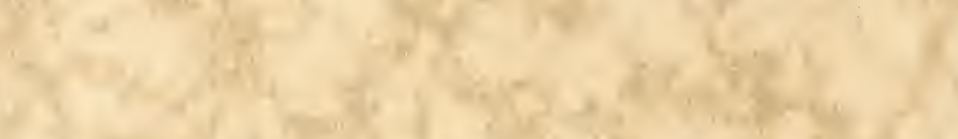

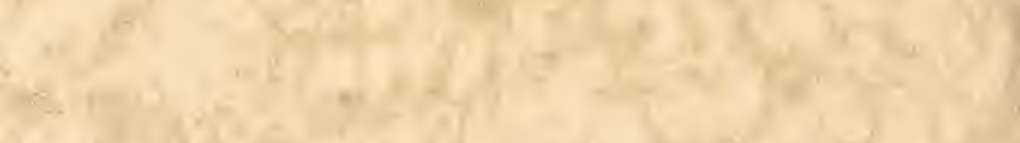

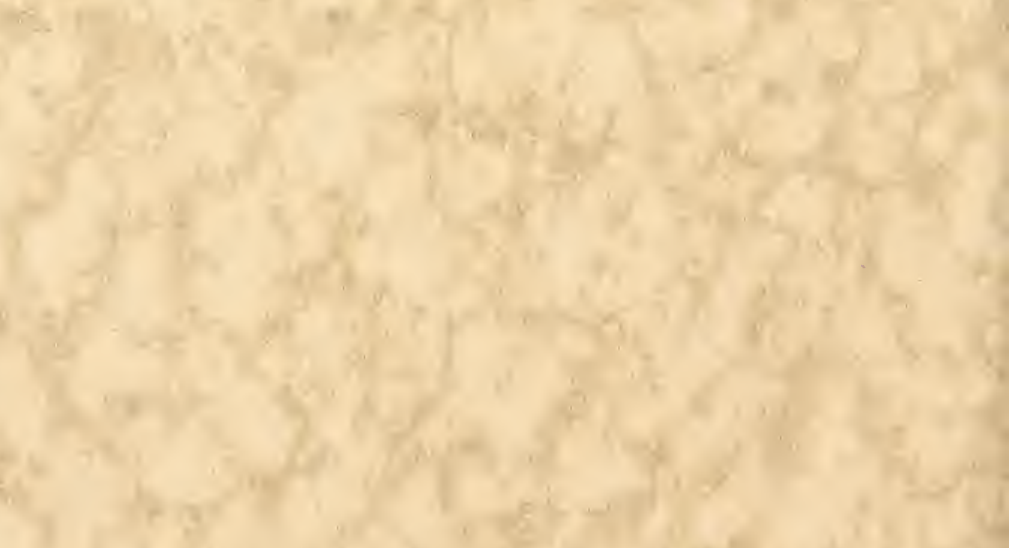
W. 